\title{
Magnetization dynamics in dysprosium orthoferrites via the inverse Faraday effect
}

\author{
C. A. Perroni and A. Liebsch \\ Institut für Festkörperforschung (IFF), Forschungszentrum Jülich, 52425 Jülich, Germany \\ (Received 24 February 2006; revised manuscript received 7 September 2006; published 31 October 2006)
}

\begin{abstract}
The ultrafast nonthermal control of magnetization has recently become feasible in canted antiferromagnets through photomagnetic instantaneous pulses [A. V. Kimel et al., Nature 435, 655 (2005)]. In this experiment, circularly polarized femtosecond laser pulses set up a strong magnetic field along the wave vector of the radiation through the inverse Faraday effect, thereby exciting nonthermally the spin dynamics of dysprosium orthoferrites. A theoretical study is performed by using a model for orthoferrites based on a general form of free energy whose parameters are extracted from experimental measurements. The magnetization dynamics is described by solving coupled sublattice Landau-Lifshitz-Gilbert equations whose damping term is associated with the scattering rate due to magnon-magnon interaction. Due to the inverse Faraday effect and the nonthermal excitation, the effect of the laser is simulated by magnetic-field Gaussian pulses with temporal width of the order of $100 \mathrm{fs}$. When the field is along the $z$ axis, a single resonance mode of the magnetization is excited. The amplitude of the magnetization and out-of-phase behavior of the oscillations for fields in the $z$ and $-z$ directions are in good agreement with the cited experiment. The analysis of the effect of the temperature shows that the magnon-magnon scattering mechanism affects the decay of the oscillations on the picosecond scale. Finally, when the field pulse is along the $x$ axis, another mode is excited, as observed in experiments. In this case, a comparison between theoretical and experimental results shows some discrepancies, the origin of which is related to the role played by anisotropies in orthoferrites.
\end{abstract}

DOI: 10.1103/PhysRevB.74.134430

PACS number(s): 78.20.Ls, 78.47.+p, 75.30.Ds

\section{INTRODUCTION}

In recent years, the need to enhance the speed of modern spin-electronic and magneto-optic devices, and to further develop the magnetic storage technology, has stimulated many studies aimed at achieving a fundamental understanding of the mechanisms of magnetization dynamics and switching. In addition to ultrafast magnetic-field pulses, which require complex devices for their generation, the spin dynamics has been induced by ultrafast optical laser pulses. Recent experiments have shown that significant demagnetization of magnetic compounds can be measured on the scale of a few hundred femtoseconds. ${ }^{1-4}$ Typically, the light is absorbed by the material, giving rise to a rapid increase of temperature responsible for the change of magnetization ${ }^{5-7}$ and spin reorientation. ${ }^{8}$ The question concerning the exact speed of the initial subpicosecond magnetization breakdown is still a subject of debate. Furthermore, the cooling time could limit the repetition frequency whose value is fundamental for actual applications. ${ }^{9}$

Recently, nonthermal ultrafast optical control of magnetization has been achieved in canted antiferromagnet samples of dysprosium orthoferrites by using circularly polarized femtosecond pulses. ${ }^{10}$ Via the inverse Faraday effect, the light excitation acts on the spins of the system as a magneticfield pulse directed along the wave vector of the radiation. This field is proportional to the intensity of light. ${ }^{11-13} \mathrm{~A}$ stimulated Raman-like coherent scattering mechanism via virtual states with strong spin-orbit coupling is responsible for the inverse Faraday effect. This effect does not rely on absorption and has its fingerprint in the fact that the helicity of the pump controls the sign of the photoinduced magnetization. Finally, this effect plays a role also in the femtosecond photomagnetic switching of spins in ferromagnetic garnet films. ${ }^{14}$
Since the manipulation of spins by means of circularly polarized laser pulses represents an advance in the field of ultrafast magnetization dynamics, we analyze thoroughly the experimental work by Kimel et al. ${ }^{10}$ and discuss its peculiarities. In this experiment, the difference between the Faraday rotations induced by right- and left-handed polarized pulses has been studied in the temperature range between 20 and $175 \mathrm{~K}$. A characteristic spin-wave mode, called quasiantiferro mode, is excited by the light pulse along the $z$ direction. When the sample is heated, the frequency of the mode oscillations increases and the amplitude decreases from the low-temperature maximum value of the order of $M_{S} / 16$, where $M_{S}$ is the saturation magnetization. We point out that there is no theoretical explanation concerning the magnitude and the temperature behavior of the oscillations. Furthermore, some aspects of the cited experiment deserve attention. Actually, in the so called $\Gamma_{4}$ phase, stable at temperatures higher than $50 \mathrm{~K}$, the oscillations have temperature-dependent frequencies in full agreement with those measured by Raman experiments. ${ }^{15,16}$ On the other hand, at temperatures below $50 \mathrm{~K}$, the frequency of the photoinduced magnetization stays constant in contrast with the results of the Raman spectra and other measurements, which signal the discontinuous transition to another phase, called $\Gamma_{1}$. Therefore, the experimental data obtained by laser pulses reflect the excitation of resonance modes characteristic of the $\Gamma_{4}$ phase even at very low temperatures. Finally, the field pulse is also directed along the $x$ axis: another spin-wave mode, called quasiferro mode, is excited and is characterized by an extraordinarily, not well understood, small amplitude.

In order to obtain a deeper understanding of the nonthermal control of magnetization, in this paper we perform simulations related to the experiment by Kimel et al. ${ }^{10}$ We have studied the magnetization dynamics employing a model for orthoferrites that was previously proposed for the analysis of 
resonance and high-frequency susceptibility. ${ }^{17}$ The parameters of the free energy, such as the symmetric and antisymmetric exchange, and the temperature-dependent anisotropy constants, are determined by using the experimental Raman spectra of Ref. 17. The dynamical behavior is described by solving two coupled sublattice nonlinear Landau-LifshitzGilbert equations through a fifth-order Runge-Kutta algorithm. The damping term in the dynamical equations is related to magnon-magnon interaction and its temperature behavior is provided by a calculation of the scattering rate in orthoferrites. ${ }^{21}$ Exploiting the inverse Faraday effect, we have analyzed the effect of Gaussian magnetic-field pulses whose time width is of the order of $100 \mathrm{fs}$. Since, in the regime considered in the experiments, the effective magnetic fields are not large if compared with exchange fields, the solution of the linearized system represents a reasonable approximation to the numerical results. Therefore, we have studied the dynamics within the linear solution after the excitation by a pulse shaped as a $\delta$ function, since the magnetic-field pulse takes place on a time scale shorter than the period of the resonance modes.

One result of this work is that, in the $\Gamma_{4}$ phase, the quasiantiferro mode of the magnetization is excited by a field pulse along the $z$ axis and the oscillations have amplitudes in agreement with experimental results. Moreover, the oscillations induced by pulses directed along the $z$ and $-z$ axis show the characteristic out-of-phase behavior. We point out that, even for the dynamics, the ratio between the antisymmetric and symmetric exchange energies is important. Furthermore, we stress that in the canted antiferromagnets, such as rareearth orthoferrites, the largest amplitudes of the oscillation are not obtained for the ferromagnetic sum vector of the sublattice magnetizations but for the antiferromagnetic difference vector. The behavior of the magnetization has been analyzed in the temperature range between 20 and $175 \mathrm{~K}$. The damping process based on magnon-magnon scattering describes the decay of the oscillations with results consistent with the experiment.

The case of the field pulse directed along the $x$ axis has been analyzed in the $\Gamma_{4}$ phase. The magnetization along the $x$ axis oscillates with the frequency of the quasiferro mode as found in the experiment. However, the calculated and the experimental amplitudes of the oscillation are different if the magnetic-field pulse along the $x$ axis has the same intensity of that along the $z$ axis. Therefore, in the comparison with experimental data, we discuss the role of anisotropies between $z$ and $x$ directions as a source of discrepancy between theory and experiment.

Finally, we consider the actual stable phase in equilibrium at low temperatures, the $\Gamma_{1}$ phase, and its resonance modes. We point out that the difference in energy between the $\Gamma_{1}$ and $\Gamma_{4}$ can be very small, so that even a small laser-heating effect could be responsible for the stabilization of the $\Gamma_{4}$ phase at very low temperatures on a picosecond time scale.

The outline of this paper is as follows. In the next section, we discuss the numerical approach for the system and the analytic solution of the linearized equations for excitation by a $\delta$-function-shaped magnetic-field pulse. Section III provides the numerical and analytical results: in the first and second subsection, the excitation due to the pulses along the

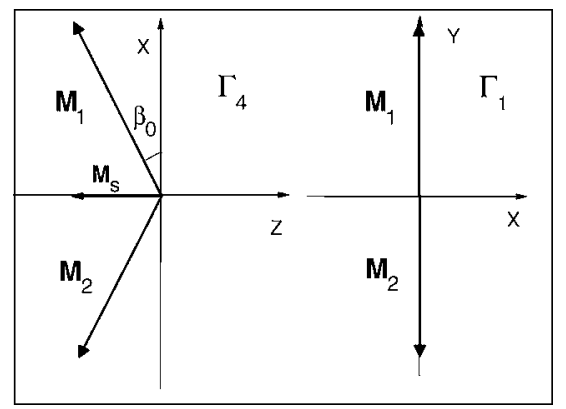

FIG. 1. The equilibrium positions of sublattice magnetization vectors for the high-temperature $\Gamma_{4}$ (ferromagnetic) and lowtemperature $\Gamma_{1}$ (antiferromagnetic) phases. Due to the ratio between the antisymmetric $D$ and symmetric $E$ exchange fields, the angle $\beta_{0}$ is actually very small.

$z$ axis and $x$ axis, respectively, is analyzed when the system at equilibrium is in the $\Gamma_{4}$ phase. In the final subsection, the effect on the dynamics of a $\Gamma_{1}$ phase stable at low temperatures is considered. Section IV provides a summary.

\section{FREE ENERGY AND DYNAMICAL EQUATIONS}

Rare-earth orthoferrites are represented by the formula $R \mathrm{FeO}_{3}$, where $R$ stands for rare earth. They have a perovskite-type structure with slight deformation from cubic to orthorombic. In many of them, the spins of iron ions are antiferromagnetically aligned through a strong superexchange interaction with a Neél transition temperature of about $700 \mathrm{~K}$. Moreover, promoted by the orthorhombic deformation, an antisymmetric exchange interaction acts between iron spins, resulting in spin-canted magnetism with a feeble saturation moment. In the case of dysprosium orthoferrites, the temperature dependence of the ferromagnetic moment is characterized by a steep rise around $50 \mathrm{~K}$ in coincidence with the stabilization of the $\Gamma_{4}$ phase. ${ }^{18}$ Actually these compounds are also known for their spin reorientation properties: continuous rotational-type (ferromagnetism present in the low-temperature phase) and, only for dysprosium, abrupt-type from $\Gamma_{1}$ (antiferromagnetic) to $\Gamma_{4}$ with increasing temperature.

We use the free energy and the dynamical equations proposed in a previous work and focus on the behavior of the magnetization in the $\Gamma_{4}$ phase. ${ }^{17}$ This description of the experimentally investigated systems turns out to be accurate since the samples are in a monodomain state. ${ }^{10}$ The static and dynamical behavior is studied within the two-sublattice model for iron spins that takes into account the two active resonance modes. This represents an excellent approximation since the remaining resonance modes are almost inactive, and their interaction with active modes is negligible. In Fig. 1 , we show a schematic representation of equilibrium positions of two-sublattice magnetization vectors for dysprosium orthoferrites. ${ }^{16}$

The normalized free energy $V=F / M_{0}$, with $M_{0}$ the modulus of the sublattice magnetization, is composed of a part $V_{\text {exc }}$ due to exchange interactions and a part $V_{\text {ani }}$ due to the anisotropy, 


$$
V=V_{\text {exc }}+V_{\text {ani }} .
$$

The free energy is expanded as a power series in the magnetization components and, in order to properly describe the $\Gamma_{4}$ phase, only quadratic terms are sufficient. The exchange energy is written as the sum of a scalar and a pseudovector part,

$$
V_{\mathrm{exc}}=E \vec{R}_{1} \cdot \vec{R}_{2}+D\left(X_{1} Z_{2}-X_{2} Z_{1}\right),
$$

where $E$ and $D$ are the symmetric and antisymmetric exchange fields, respectively, $\vec{R}_{1}=\vec{M}_{1} / M_{0} \equiv\left(X_{1}, Y_{1}, Z_{1}\right)$, and $\vec{R}_{2}=\vec{M}_{2} / M_{0} \equiv\left(X_{2}, Y_{2}, Z_{2}\right)$. The anisotropic energy is

$$
V_{\text {ani }}=-A_{x x}\left(X_{1}^{2}+X_{2}^{2}\right)-A_{z z}\left(Z_{1}^{2}+Z_{2}^{2}\right) \text {. }
$$

The exchange field $E$ is quite large compared to the other terms. It is related to the exchange spin-spin interaction $J$ via the equation $E=12 \mathrm{JS} / g \mu_{B}$, with $S=5 / 2$ the spin of iron ions, $g=2$, and $\mu_{B}$ the Bohr magneton. Since $J$ is of the order of $20 \mathrm{~cm}^{-1}, E$ is approximately $6.4 \times 10^{6}$ Oe. ${ }^{16}$ The field $D$ is related to the antisymmetric exchange energy $d$ through the relation $D=6 d S / g \mu_{B}$ and is of the order of $1.4 \times 10^{5} \mathrm{Oe}(d$ is about $0.88 \mathrm{~cm}^{-1}$ ). The anisotropy constants $A_{x x}$ and $A_{z z}$ depend on temperature and are of the order of hundreds of Oersted.

Using the energy given in Eq. (1), the equilibrium position can be derived for the $\Gamma_{4}$ phase. This is shown in Fig. 1, where the small canting angle $\beta_{0}$ is determined by the equation

$$
\tan \left(2 \beta_{0}\right)=\frac{D}{E+A_{x x}-A_{z z}} \simeq \frac{D}{E}=0.022 .
$$

Hence the magnetization $M_{S}=\left|\vec{M}_{1}+\vec{M}_{2}\right|=2 M_{0} \sin \left(\beta_{0}\right)$ $\sim 0.022 M_{0}$ is two orders of magnitude less than $M_{0}$.

The equilibrium position of $\vec{M}_{1}$ and $\vec{M}_{2}$ represents a stationary solution of the nonlinear Landau-Lifshitz-Gilbert equations

$$
\begin{aligned}
& \frac{d \vec{R}_{1}}{d t}=-\gamma \vec{R}_{1} \wedge\left[\vec{H}(t)-\vec{\nabla}_{1} V\right]+\alpha \vec{R}_{1} \wedge \frac{d \vec{R}_{1}}{d t}, \\
& \frac{d \vec{R}_{2}}{d t}=-\gamma \vec{R}_{2} \wedge\left[\vec{H}(t)-\vec{\nabla}_{2} V\right]+\alpha \vec{R}_{2} \wedge \frac{d \vec{R}_{2}}{d t},
\end{aligned}
$$

where $\gamma=17.6 \mathrm{MHz} / \mathrm{Oe}$ is the gyroscopic ratio, $\vec{\nabla}_{1}$ and $\vec{\nabla}_{2}$ are gradients with respect to $\vec{R}_{1}$ and $\vec{R}_{2}$, respectively, and $V$ is the energy in Eq. (1). Clearly the dynamical equations satisfy the following constraints: $X_{1}^{2}+Y_{1}^{2}+Z_{1}^{2}=1$ and $X_{2}^{2}+Y_{2}^{2}+Z_{2}^{2}=1$.

The quantity $\vec{H}(t)$ in Eqs. (5) and (6) is the timedependent magnetic field simulating the effect of laser pulses due to the inverse Faraday effect. ${ }^{11,12}$ The ultrafast magnetic field is optically generated by a stimulated Raman-like coherent scattering mechanism via virtual states with strong spin-orbit coupling [see Eq. (25) of Ref. 13]. Within this second-order process, the intermediate excited states are coupled to states in the ground-state manifold via dipole matrix elements and the strength of the laser electric field. The effective magnetic field couples to spins of the ground-state manifold and is proportional to the modulus square of the electric field, i.e., to the intensity of the laser pulse. In the experiment by Kimel et al., ${ }^{10}$ the magnetic-field pulse is of the order of fractions of Tesla with a time width of $100 \mathrm{fs}$. In the numerical simulations, we consider a pulse directed along the propagation direction of the light with a Gaussian shape,

$$
\vec{H}(t)=\hat{k} \frac{F_{0}}{\sqrt{\pi} \tau_{p}} \exp \left[-\left(t / \tau_{p}\right)^{2}\right],
$$

where $\hat{k}$ defines the direction of the light wave vector and $\tau_{p}$ indicates approximately the duration of the pulse. We stress that this field is different from the transverse circularly polarized magnetic field, which is present together with the electric field of the laser pulse. ${ }^{19}$

Finally, in Eqs. (5) and (6), $\alpha$ is the Gilbert constant. Scattering mechanisms due to electrons are negligible since rare-earth orthoferrites are insulating. These systems are different from itinerant ferromagnetic compounds where the behavior of the conduction electrons strongly influences the relaxation of the magnetization on the femtosecond time scale. In orthoferrites, the quantity $\alpha$ takes into account the damping of the oscillations due to the magnon-magnon scattering and to the interaction of magnons with dysprosium spins and phonons. We notice that the scattering via dysprosium spins should be larger at very low temperatures where dysprosium ions tend to order. Moreover, the phononmagnon scattering should be effective only on the nanosecond time scale. ${ }^{14,20}$ It is the magnon-magnon interaction that provides the relevant source of scattering on the picosecond time scale in the monodomain samples.

\section{A. Solution of the linearized system}

In this subsection, we consider the solution determined by linearizing Eqs. (5) and (6) and study the excitation of this linear system due to a magnetic-field pulse shaped as a $\delta$ function. This is reasonable since, in the regime considered in the experiments, the effective magnetic fields are not large if compared with exchange fields, and the temporal width of the pulse is much smaller than the periods of the resonance modes.

In order to take into account small deviations from the equilibrium, the standard approach is to consider two separate coordinate systems, $\left(S_{1}, T_{1}, Y_{1}\right)$ and $\left(S_{2}, T_{2}, Y_{2}\right)$, which describe the dynamics of $\vec{M}_{1}$ and $\vec{M}_{2}$, respectively. ${ }^{17}$ The variables $S_{1}$ and $S_{2}$ are chosen in order to coincide with the equilibrium positions of $\vec{M}_{1}$ and $\vec{M}_{2}$, respectively, so that

$$
\begin{gathered}
S_{1}=\sin \left(\beta_{0}\right) Z_{1}+\cos \left(\beta_{0}\right) X_{1}, \\
T_{1}=-\cos \left(\beta_{0}\right) Z_{1}+\sin \left(\beta_{0}\right) X_{1},
\end{gathered}
$$

and

$$
\begin{aligned}
& S_{2}=\sin \left(\beta_{0}\right) Z_{2}-\cos \left(\beta_{0}\right) X_{2}, \\
& T_{1}=\cos \left(\beta_{0}\right) Z_{2}+\sin \left(\beta_{0}\right) X_{2} .
\end{aligned}
$$

By linearizing the system, we obtain the frequencies of two modes, the quasi-antiferro $\omega_{\mathrm{AFM}}$ and the quasiferro $\omega_{\mathrm{FM}}$ 
modes, involving cooperative motions of spins of the two sublattices. The energy of the quasi-antiferro and quasiferro modes is of the order of several $\mathrm{cm}^{-1}$.

The first mode is characterized by the frequency

$$
\frac{\omega_{\mathrm{AFM}}^{2}}{\gamma^{2}}=4 E A_{x x}+4 A_{x x}\left(A_{x x}-A_{z z}\right)+D^{2} .
$$

The dynamic in this mode shows the following behavior: $\Delta X_{1}(t)=-\Delta X_{2}(t), \quad \Delta Y_{1}(t)=-\Delta Y_{2}(t), \quad$ and $\quad \Delta Z_{1}(t)=\Delta Z_{2}(t)$, where $\Delta W_{i}(t)=W_{i}(t)-W_{i}^{\text {eq }}$, with $W=X, Y, Z, i=1,2$, and $W_{i}^{\text {eq }}$ corresponding to the equilibrium position shown in Fig. 1. Thus, the only ferromagnetic component different from zero is the magnetization along the $z$ axis with respect to the equilibrium, $\Delta M_{Z}=M_{0} \Delta \tilde{M}_{Z}$, where $\Delta \tilde{M}_{Z}$ is defined by

$$
\Delta \tilde{M}_{Z}=\Delta Z_{1}(t)+\Delta Z_{2}(t)=Z_{1}(t)+Z_{2}(t)-\left(Z_{1}^{\mathrm{eq}}+Z_{2}^{\mathrm{eq}}\right) .
$$

Since the net spin does not reflect the motions of the sublattice spins, this mode is called quasi-antiferromagnetic.

The second mode, with frequency

$$
\frac{\omega_{\mathrm{FM}}^{2}}{\gamma^{2}}=4 E\left(A_{x x}-A_{z z}\right)+4 A_{x x}\left(A_{x x}-A_{z z}\right),
$$

is characterized by $\Delta X_{1}(t)=\Delta X_{2}(t), \Delta Y_{1}(t)=\Delta Y_{2}(t)$, and $\Delta Z_{1}(t)=-\Delta Z_{2}(t)$. Since the net spin executes the same rocking behavior as the sublattice spins, this mode is called quasiferromagnetic.

Equations (12) and (14) relate the mode frequencies to the model parameters. The experimental mode frequencies are dependent on temperature. The exchange fields are assumed constant in temperature at the values given above, since they represent the highest energy scales. Using Eqs. (12) and (14) and the experimentally measured Raman spectra of Ref. 17, we derive the temperature-dependent anisotropy parameters. The quasi-antiferro mode increases linearly as a function of temperature in the $\Gamma_{4}$ phase: from $150 \mathrm{GHz}$ at $50 \mathrm{~K}$ to $450 \mathrm{GHz}$ at about $200 \mathrm{~K}$, while the quasiferro mode stays constant at about $375 \mathrm{GHz}$. Therefore, the anisotropic terms change upon heating the sample: $A_{x x}$ varies from about $-640 \mathrm{Oe}$ at $T=50 \mathrm{~K}$ to about $200 \mathrm{Oe}$ at $T=200 \mathrm{~K}, A_{z z}$ from -1540 to -700 Oe in the same temperature range, with approximately fixed $A_{x x}-A_{z z}=900 \mathrm{Oe}$. The knowledge of the frequencies is important also to determine the value of the damping constant in the dynamical equations (5) and (6). Indeed, if $\omega_{0}$ is the temperature-dependent frequency of one of the modes, the damping constant $\alpha$ could be related to the damping rate $1 / \tau_{0}$ by the relation $1 / \tau_{0}=\omega_{0} \alpha$. At the picosecond scale, the dominant spin-wave damping is due to fourmagnon scattering. By using many-body perturbation theory, the rate for spin wave at zero wave vector is estimated to be $1 / \tau_{0} \sim 2.66 \times 10^{4} \mathrm{~T}^{2} \mathrm{~s}^{-1}$, with $T$ temperature in units of degrees Kelvin. ${ }^{21}$ The scattering rate grows quadratically in temperature, while the frequency of the quasi-antiferro mode is an increasing linear function of the temperature. ${ }^{16}$ The quantity $\alpha$ gets larger with temperature since the most important contribution is given by $1 / \tau_{0}$. Actually, the values of $\alpha$ corresponding to the quasi-antiferro mode range from about $0.4 \times 10^{-4}$ at $T=50 \mathrm{~K}$ to $3 \times 10^{-4}$ at $T=200 \mathrm{~K}$. The smallness of $\alpha$ implies that the oscillations of the magnetization are not strongly damped with increasing temperature. Within the model, temperature affects the mode frequencies via the anisotropy constants and the damping constant.

With the determination of $\alpha$, all the parameters appearing in Eqs. (5) and (6) can be evaluated. We stress that the timedependent effective magnetic field induced by light can be assumed uniform in space. This is the reason why it couples only to spin waves with zero wave vector (the quasiferro and quasi-antiferro modes discussed above). The spin waves with wave vectors different from zero can be thermally excited decreasing the static value of the magnetization. In the considered range of temperature, as shown in experiments, ${ }^{18}$ this decrease is small. With increasing temperature, the role of $\alpha$, the damping term of the spin waves with zero wave vector, is fundamental in affecting the behavior of the amplitude of the magnetization. This justifies the macrospin approximation used in our approach.

It is useful to consider a magnetic field pulse shaped as a $\delta$ function,

$$
\vec{H}(t)=\hat{k} F_{0} \delta(t) .
$$

Since the pulse is instantaneous, it provides an initial condition to the dynamics described by the linearized equations of motions. We have analyzed two cases: the field along the $z$ and $x$ axes, since these are prominent for the experiment that we want to discuss.

For the field along the $z$ axis, starting from the equilibrium position at $t=0^{-}$, we find at $t=0^{+}$

$$
\begin{gathered}
\Delta X_{1}\left(0^{+}\right)=-\Delta X_{2}\left(0^{+}\right)=\cos \left(\beta_{0}\right)\left[\cos \left(\gamma F_{0}\right)-1\right], \\
\Delta Y_{1}\left(0^{+}\right)=-\Delta Y_{2}\left(0^{+}\right)=\cos \left(\beta_{0}\right) \sin \left(\gamma F_{0}\right), \\
\Delta Z_{1}\left(0^{+}\right)=\Delta Z_{2}\left(0^{+}\right)=0 .
\end{gathered}
$$

Therefore, this pulse will excite only the quasi-antiferro mode.

The field along the $x$ axis yields at $t=0^{+}$

$$
\begin{gathered}
\Delta X_{1}\left(0^{+}\right)=\Delta X_{2}\left(0^{+}\right)=0, \\
\Delta Y_{1}\left(0^{+}\right)=\Delta Y_{2}\left(0^{+}\right)=-\sin \left(\beta_{0}\right) \sin \left(\gamma F_{0}\right), \\
\Delta Z_{1}\left(0^{+}\right)=-\Delta Z_{2}\left(0^{+}\right)=\sin \left(\beta_{0}\right)\left[\cos \left(\gamma F_{0}\right)-1\right] .
\end{gathered}
$$

Thus the system will be excited in the quasiferro mode.

As the experimental field magnitude is lower than the exchange fields, we study the subsequent dynamical evolution within the linear approximation. ${ }^{17}$ Within a relaxation time approximation, we include the damping time $\tau$ describing the decay toward the equilibrium position. ${ }^{22}$

From the solution of the linear system in the case of a pulse along the $z$ axis, we get the evolution of the quasiantiferro mode. The only ferromagnetic component, defined in Eq. (13), is 


$$
\begin{aligned}
\Delta \tilde{M}_{Z}= & e^{-t / \tau} \frac{\cos \left(\beta_{0}\right)}{2} \sin \left(2 \beta_{0}\right) \sin ^{2}\left(\frac{\gamma F_{0}}{2}\right) \cos \left(\omega_{\mathrm{AFM}} t\right) \\
& +2 e^{-t / \tau} R \cos ^{2}\left(\beta_{0}\right) \sin \left(\gamma F_{0}\right) \sin \left(\omega_{\mathrm{AFM}} t\right) \\
& +2 e^{-t / \tau} \sin \beta_{0} \cos ^{2}\left(\beta_{0}\right)\left[\cos \left(\gamma F_{0}\right)-1\right] .
\end{aligned}
$$

We notice in Eq. (22) that the even terms in the magnetic field are at least two orders of magnitude less than the odd term.

Along the other two directions, there are the antiferromagnetic components along the $x$ axis (with respect to the equilibrium), $\Delta A_{X}=M_{0} \Delta \widetilde{A}_{X}$, with $\Delta \widetilde{A}_{X}$ defined as

$$
\Delta \tilde{A}_{X}=\Delta X_{1}(t)-\Delta X_{2}(t)=X_{1}(t)-X_{2}(t)-\left(X_{1}^{\mathrm{eq}}-X_{2}^{\mathrm{eq}}\right),
$$

and the antiferromagnetic component along the $y$ axis (with respect to the equilibrium), $\Delta A_{Y}=M_{0} \Delta \tilde{A}_{Y}$, with $\Delta \tilde{A}_{Y}$,

$$
\Delta \tilde{A}_{Y}=\Delta Y_{1}(t)-\Delta Y_{2}(t)=Y_{1}(t)-Y_{2}(t)-\left(Y_{1}^{\mathrm{eq}}-Y_{2}^{\mathrm{eq}}\right) .
$$

Using again the linearized form of Eqs. (5) and (6), we obtain

$$
\begin{aligned}
\Delta \tilde{A}_{X}= & -e^{-t / \tau} \sin \left(2 \beta_{0}\right) \sin \left(\beta_{0}\right) \sin ^{2}\left(\frac{\gamma F_{0}}{2}\right) \cos \left(\omega_{\mathrm{AFM}} t\right) \\
& -e^{-t / \tau} R \sin \left(2 \beta_{0}\right) \sin \left(\gamma F_{0}\right) \sin \left(\omega_{\mathrm{AFM}} t\right) \\
& +2 e^{-t / \tau} \cos \beta_{0} \cos ^{2}\left(\beta_{0}\right)\left[\cos \left(\gamma F_{0}\right)-1\right]
\end{aligned}
$$

and

$$
\begin{aligned}
\Delta \tilde{A}_{Y}= & -e^{-t / \tau} \frac{1}{R} \sin \left(2 \beta_{0}\right) \sin ^{2}\left(\frac{\gamma F_{0}}{2}\right) \sin \left(\omega_{\mathrm{AFM}} t\right) \\
& +2 e^{-t / \tau} \cos \left(\beta_{0}\right) \sin \left(\gamma F_{0}\right) \cos \left(\omega_{\mathrm{AFM}} t\right) .
\end{aligned}
$$

The components specified in Eqs. (22), (25), and (26) are determined by the mode frequency $\omega_{\mathrm{AFM}}$, the angle $\beta_{0}$, and the quantity $R$, which depends on the model parameters in the form

$$
R=\sqrt{\frac{2 A_{x x}+D^{2} / 2 E}{2 E}} .
$$

At intermediate temperatures ( $A_{x x}$ very small), one gets $R$ $\simeq D / 2 E$

When the pulse is directed along the $x$ direction, the quasiferro mode is excited. The components of the magnetization different from zero are those along the $x$ and $y$ axes. In particular we consider the magnetization along the $x$ axis (with respect to equilibrium), $\Delta M_{X}=M_{0} \Delta \tilde{M}_{X}$, where $\Delta \tilde{M}_{X}$ is defined as

$$
\Delta \tilde{M}_{X}=\Delta X_{1}(t)+\Delta X_{2}(t) .
$$

From the linearized dynamical equations, this quantity is calculated as

$$
\Delta \tilde{M}_{X}=e^{-t / \tau} \sqrt{\frac{4 E}{A_{x x}-A_{z z}}} \sin ^{2}\left(\beta_{0}\right) \sin \left(\gamma F_{0}\right) \sin \left(\omega_{\mathrm{FM}} t\right) .
$$

\section{NUMERICAL AND ANALYTICAL RESULTS}

Using the procedure outlined in the previous section, all the parameters appearing in the dynamical equations (5) and (6) can be determined. These nonlinear equations are numerically integrated through a fifth-order Runge-Kutta algorithm. ${ }^{23}$ The experimental laser pulses along the $z$ axis are estimated to be equivalent to magnetic fields with an amplitude of $0.3 \mathrm{~T}$ and a full width at half-maximum of about $200 \mathrm{fs} .{ }^{10}$ Therefore, we consider Gaussian fields of the form given in Eq. (7), with $F_{0}=\sqrt{\pi} \tau_{p} \operatorname{amp}(H), \tau_{p}=200 \mathrm{fs}$, and $\operatorname{amp}(H)$ typically $3000 \mathrm{Oe}$. This value of the effective field is small when compared with the exchange fields, so that effects due to the nonlinearity of the equations are negligible. Since the time width of the pulse is small on the scale of the mode periods, the results are not strongly dependent on $\tau_{p}$. The linear solution with a $\delta$ pulse represents a good approximation, provides the right orders of magnitude, but does not reproduce exactly the numerical results. Therefore, in all the following figures we plot the results obtained by the numerical integration.

In the first and second subsections, we discuss the effects of a Gaussian pulse along the $z$ and $x$ directions, respectively, when the system at equilibrium is in the $\Gamma_{4}$ phase. In the final one we briefly analyze the resonance modes and magnetization dynamics if the $\Gamma_{1}$ phase is stable at low temperatures.

\section{A. Pulse along the $z$ direction}

As illustrated in Ref. 12, pulses along the $z$ and $-z$ directions excite the quasi-antiferro mode. Using the numerical procedure discussed in the preceding section, we have calculated the oscillating behavior of the ferromagnetic vector along $z$ focusing on $\Delta M_{Z}$ at $T=95 \mathrm{~K}$. As shown in Fig. 2(a), the magnetization has a sinelike behavior with weak damping on a picosecond scale. In addition, opposite fields give rise to out-of-phase oscillations in agreement with the experimental data. We notice that after $50 \mathrm{ps}$, the amplitude is about half of the initial one, a value that is compatible with results reported in Fig. 1 of Ref. 12. This suggests that the damping term associated with magnon-magnon scattering provides a reasonable description of the reduction of the amplitude as a function of temperature.

Comparing the experimental data shown in Fig. 1 of Ref. 12 with the theoretical results shown in Fig. 2(a) of this paper, we point out similarities and differences. Indeed, on the scale of the initial pulse width, there is a strong enhancement of the experimental Faraday rotation, probably due to the interference between pump and probe pulses. After this transient, the oscillations induced by pulses with opposite helicities show similar amplitudes in time. In contrast with theoretical results, the equilibrium point of the Faraday rotations shows a decay and its behavior is different for left- and right-handed polarized pulses. Only later do the oscillations 

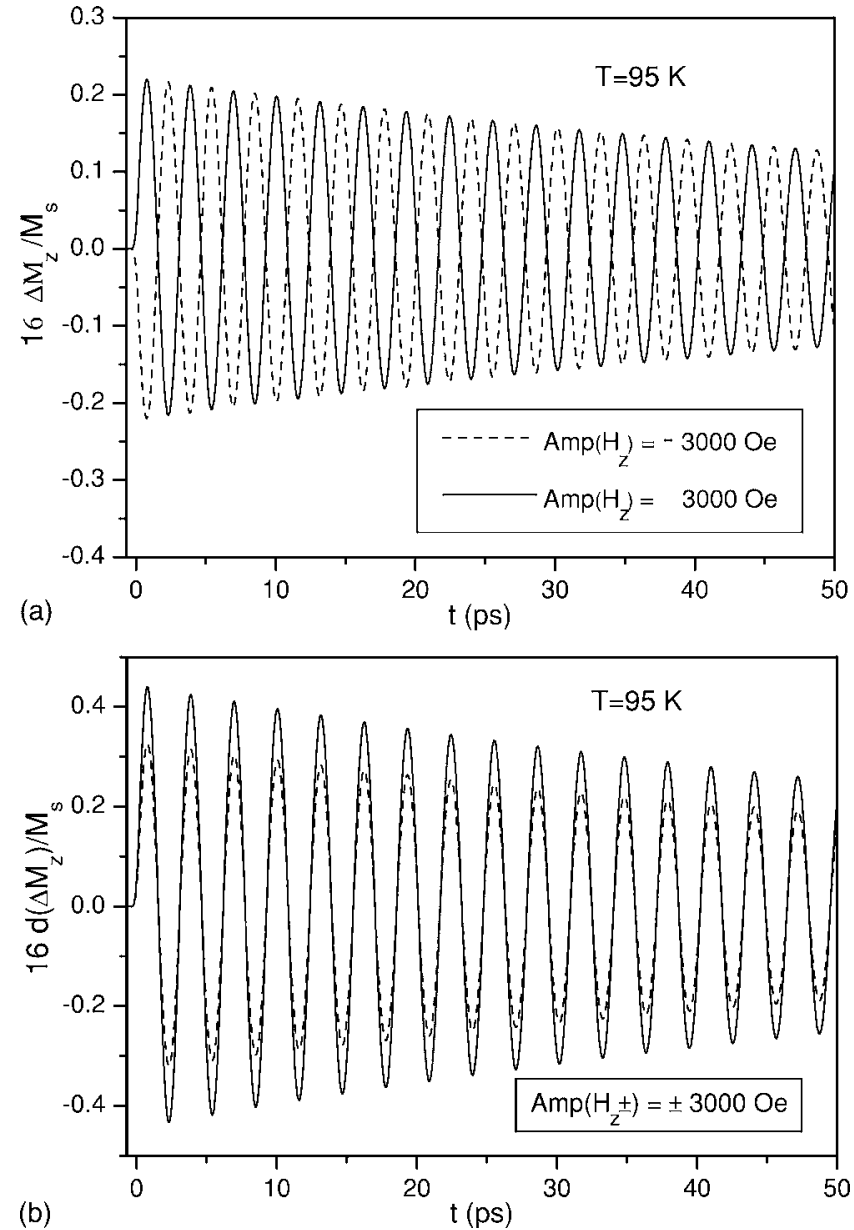

FIG. 2. (a) The variation $\Delta M_{Z}$ of the magnetization along the $z$ axis with respect to the equilibrium value in units of $M_{S} / 16$ as a function of time for two opposite amplitudes. (b) The difference $d\left(\Delta M_{Z}\right)$ in units of $M_{S} / 16$ between the magnetizations obtained upon excitation by opposite fields along the $z$ direction as a function of time. The dashed line includes the effect of the anisotropy that will be introduced in the next subsection.

tend toward a common equilibrium point that is different, however, from that before the pulse excitation. If the shift of the equilibrium positions is caused by a change of the anisotropy constants due to an intrinsic photomagnetic effect, then the amplitude of the oscillations should be different for the two helicities, as reported in a recent experiment. ${ }^{14}$ Since this is not the case in dysprosium orthoferrites, the change in the orientation equilibrium is more probably associated with other effects.

In Fig. 2(b), we focus on the related quantity $d\left(\Delta M_{Z}\right)$, defined as

$$
d\left(\Delta M_{Z}\right)=\Delta M_{Z}(H+)-\Delta M_{Z}(H-),
$$

with $H+$ and $H-$ indicating positive and negative amplitudes, respectively. As reported in the experimental work, ${ }^{10}$ this quantity is less dependent on initial effects. Just after the transient induced by the pulse, this quantity has an amplitude of the order of $M_{S} / 16$, in close agreement with the calculated data reported in Fig. 2(b). It is worthwhile understanding the

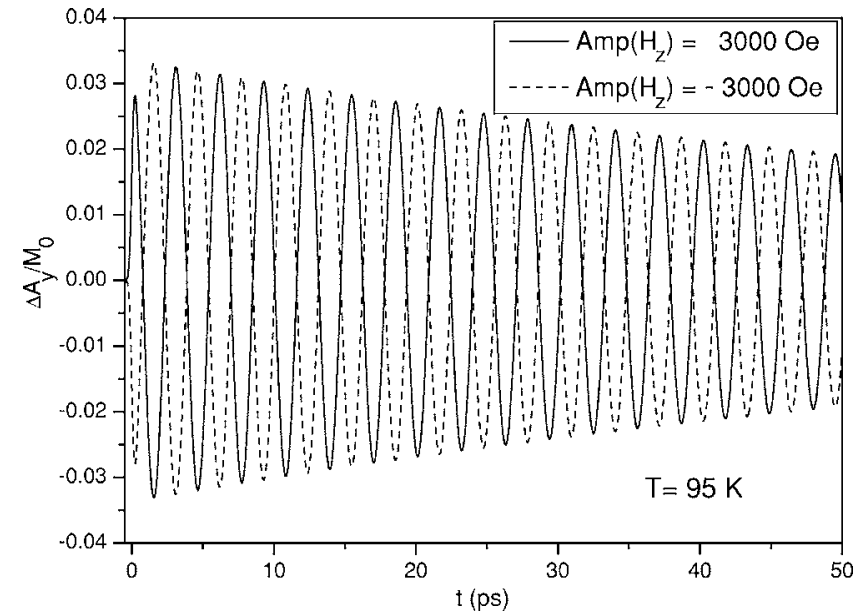

FIG. 3. The variation of the antiferromagnetic vector along the $y$ axis $\Delta A_{Y}$ with respect to $M_{0}$ as a function of time for two opposite amplitudes.

order of magnitude of this amplitude by exploiting the result of the previous section for the $\delta$ pulse. From Eq. (22), we get for short times $\left(t \ll \tau_{2}\right)$

$$
\Delta M_{Z} \simeq 2 M_{0} R \sin \left(\gamma F_{0}\right) \sin \left(\omega_{\mathrm{AFM}} t\right),
$$

with $R$ given in Eq. (27). The amplitude is determined by the term $R$ depending on the model parameters and by $\sin \left(\gamma F_{0}\right)$ related to the pulse intensity. Using the experimental data, the impulse $F_{0}$ is estimated to be of the order of 3000 $\times 400$ Oe fs. This implies that $\gamma F_{0}$ is of the same order as the angle $\beta_{0}$ responsible for the canted antiferromagnetism: $\sin \left(\gamma F_{0}\right) \sim \gamma F_{0} \sim 2 \beta_{0}$. Thus, we have

$$
\Delta M_{Z} / M_{S} \simeq 2 R \sin \left(\omega_{\mathrm{AFM}} t\right)
$$

If we consider $R \simeq D / 2 E$, we obtain the order of magnitude $16 d\left(\Delta M_{Z}\right) / M_{S} \sim 16 \times 2 \times 0.02=0.64$ not so far from the experiment. Hence the ratio $D / E$ is fundamental not only for the equilibrium configuration, but also for the magnetization dynamics in the canted antiferromagnet.

Within the theoretical approach, the dynamics of the antiferromagnetic vectors can be easily obtained, but these quantities have not been measured in the experimental work. ${ }^{10}$ Due to the fact that the equilibrium position corresponds to a maximum of $A_{X}=M_{0}\left(X_{1}-X_{2}\right)$, the temporal evolution of $\Delta A_{X}=M_{0} \Delta \widetilde{A}_{X}$, with $\Delta \widetilde{A}_{X}$ defined in Eq. (23), is characterized by a very small amplitude. From Eq. (25) one can deduce that the amplitude of $\Delta A_{X}$ should be at least an order of magnitude smaller than that of $\Delta M_{Z}$.

In Fig. 3, we show the results of the numerical calculation for the quantity $\Delta A_{Y}=M_{0} \Delta \tilde{A}_{Y}$, where $\Delta \tilde{A}_{Y}$ is defined in Eq. (24). We point out that now the amplitude is an order of magnitude larger than that of $\Delta M_{Z}$ and the response is more sensitive to the pulse. Actually this is due to the fact that the dynamics of the components along the $y$ axis is strongly influenced by the highest energy scale $E .{ }^{17}$ From Eq. (26) we obtain a rough estimate for short times $\left(t \ll \tau_{2}\right)$, 


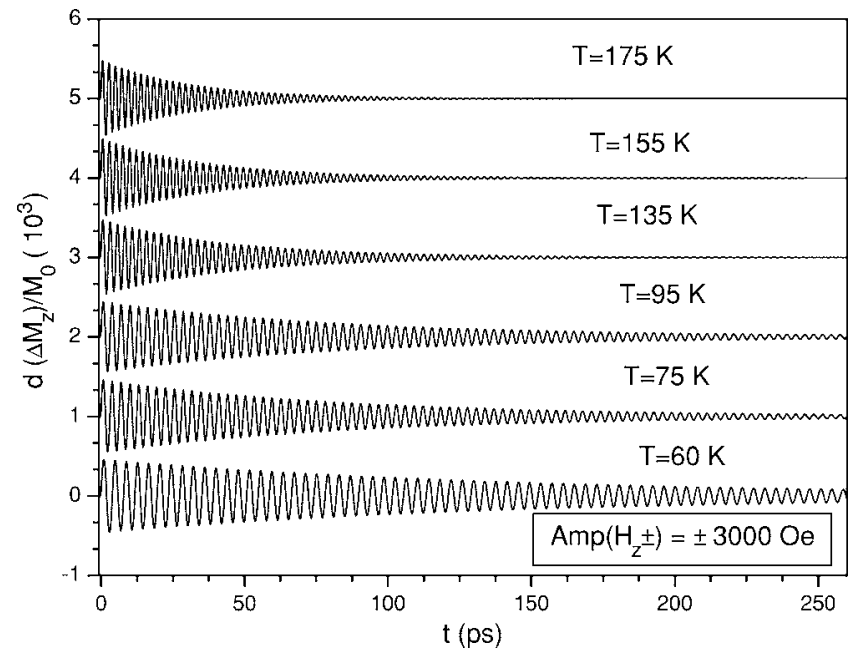

FIG. 4. The difference between the magnetizations along $z$ for opposite fields as a function of temperature. Every new curve is shifted from the previous one along the vertical axis over 1 .

$$
\Delta A_{Y} \simeq 2 M_{0} \sin \left(\gamma F_{0}\right) \cos \left(\omega_{\mathrm{AFM}} t\right) .
$$

Therefore, the amplitude is $2 \sin \left(\gamma F_{0}\right) M_{0} \sim 2 \beta_{0} M_{0}$ $\sim 0.022 M_{0}=M_{S}$. Finally, we notice that, after $50 \mathrm{ps,}$, the damping acts in the same way as for the ferromagnetic vector along the $z$ direction, causing a reduction of about half of the initial amplitude.

In order to make contact with the experimental measurements, it is interesting to analyze the behavior of the magnetization dynamics at different temperatures. As reported in Fig. 3 of the paper by Kimel et al., ${ }^{10}$ upon heating the sample, the frequency of the oscillations increases and the amplitude decreases. The oscillations have temperaturedependent frequencies that are very close to those measured by Raman experiments at temperatures higher than $50 \mathrm{~K}$. In Fig. 4 of this paper, the calculated $d\left(\Delta M_{Z}\right)$ is shown. We find agreement with experimental decay of amplitudes and behavior of the frequency upon increasing the temperature for times after the transient. Within the theoretical approach, the temperature dependence of the anisotropy constants $A_{x x}$ and $A_{z z}$ affects the frequency of the modes, but is not so important in the change of the amplitude after the laser transient. The decrease of the magnetization seen at a fixed time (of the order of tens of picoseconds) for different temperatures is dominated by the increase of the damping constant $\alpha$ in temperature. Due to the agreement with experiment, the estimate of the damping constant derived on the basis of the magnonmagnon scattering is reliable.

The results of Fig. 4 show the magnetization normalized by the sublattice magnetization modulus $M_{0}$. We point out that, in principle, $M_{0}$ can vary as a function of temperature. However, the inclusion of the temperature dependence of $M_{0}=M_{z} /\left[2 \sin \left(\beta_{0}\right)\right]$ is not easy, since only the magnetization $M_{z}$ is typically experimentally available. In any case, even if the angle $\beta_{0}$ is assumed fixed, the change of $M_{z}$ is small in the considered temperature range. ${ }^{18}$

Before closing this section, we focus on the dependence of the magnetic response on the amplitude of the pulse. As

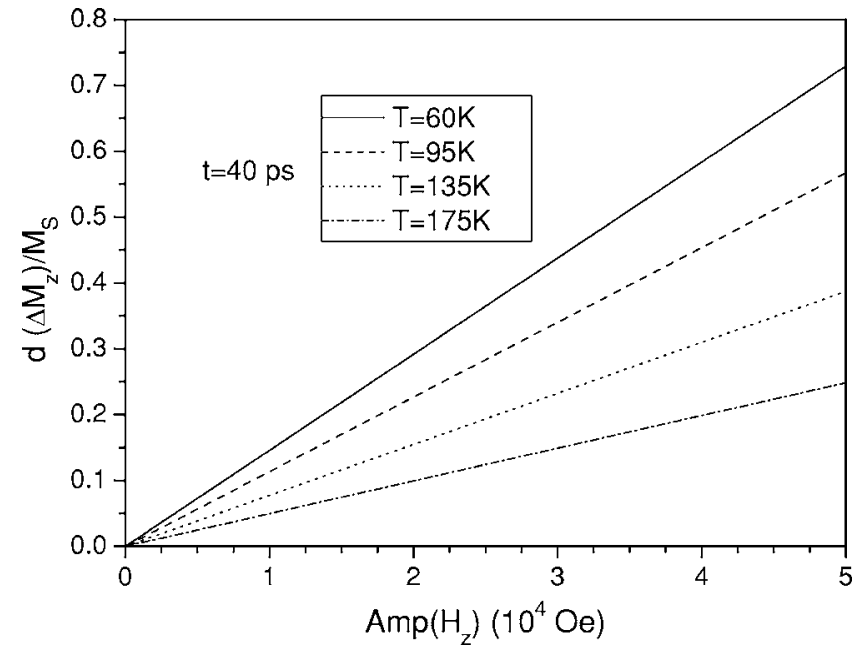

FIG. 5. Amplitude of the spin oscillations as a function of the amplitude of the magnetic-field pulse for different temperatures.

inferred by Eq. (31), for the effective magnetic pulses used in the experiments, the ferromagnetic vector is proportional to $F_{0}$, i.e., to the intensity of the light pulse used in the inverse Faraday effect. In Fig. 5, we plot the numerical results for the amplitude of the magnetization at $t=40 \mathrm{ps}$, i.e., after about 10 periods as in the experimental data. ${ }^{24}$ We find the expected linear behavior as a function of the amplitude of the pulse. The value obtained at $5 \mathrm{~T}$ for $T=60 \mathrm{~K}$ is not far from unity. Thus, the saturation of the magnetization along $z$ is reached for this high intensity, in agreement with the extrapolation of the experimental data in the inset of Fig. 2 of Ref. 12.

Summarizing, in this subsection we have focused on the magnetization dynamics in the $\Gamma_{4}$ phase when the field pulse is directed along the $z$ axis. We have analyzed the amplitude and the decay of the oscillations as a function of temperature finding agreement with experimental data for times longer than the initial transient. Actually, on the time scale of the initial pulse width, discrepancies with experiments appear. These could be due to a small but unavoidable laser-heating effect. Even more important might be the role played by birefringence. ${ }^{25}$ It is well known that in rare-earth orthoferrites, optical birefringence is not negligible. ${ }^{26,27}$ It has been experimentally checked that the main source of the asymmetry on the short time scale and its temperature dependence are due to birefringence. ${ }^{24}$ Furthermore, in the presence of birefringence, the comparison between theory and experiment is complicated by the fact that the link between the Faraday rotation (measured quantity) and the magnetization (calculated quantity) is not so direct.

\section{B. Pulse along the $x$ direction: Role of anisotropy}

In this subsection, we analyze the effects of the excitation due to the pulse along the $x$ axis.

The fields along the $x$ direction excite another mode. As shown in Eq. (29), $\Delta \tilde{M}_{X}$ oscillates with the frequency of the quasiferro mode, in agreement with experiment. On the other hand, the magnitude of the magnetic response along $x$ is of 
the same order as that obtained along the $z$ axis with a pulse of equal amplitude. Even if the torque exerted by the field along $x$ is tiny, the subsequent temporal evolution, in particular that of the variables $T_{1}$ and $T_{2}$, is able to give a nonnegligible amplitude to the response along $x$. This is in contrast with the experimental results, which show that the Faraday rotation along $x$ is at least an order of magnitude smaller than that along the $z$ axis. ${ }^{10}$ Also, as shown in Fig. 3 of Ref. 12, it does not show any temperature dependence following the behavior of the frequency of the quasiferro mode, in striking contrast with the case of a pulse along the $z$ axis.

In order to properly compare the experimental and theoretical results, we should take into account several effects in orthoferrites. ${ }^{25}$ First of all, the experimental estimate of the effective magnetic-field pulse due to the inverse Faraday effect is available only when the pulse is along the $z$ axis. Moreover, due to optical birefringence, the experimental Faraday rotations along the $z$ and $x$ axes can be different, even if the oscillations of the magnetizations are of the same order. Since the Faraday rotation along the $x$ direction is very small in comparison with that along the $z$ axis, part of the effect could also be associated with the anisotropy of the magnetooptical susceptibility. Indeed, the effective magnetic field generated via the inverse Faraday effect can be different for the two orthogonal orientations. Finally, there is another source of anisotropy: the field along the $z$ axis can show a renormalization of the coupling to the system that is different from that along the $x$ axis.

In order to elucidate this last point, we have included in our model the anisotropy induced by the dysprosium ions. As a result, a modification of the coupling of the magnetic field to the iron ions takes place. According to a simple scheme proposed long ago by Zvezdin and Matveev ${ }^{28}$ the coupling to the field along the $z$ direction is reduced by the factor 1 $+\eta_{z} \simeq 0.74$ [see in Fig. 2(b) the effect of this reduction]. Instead, the reduction $1+\eta_{x}$ of the coupling along the $x$ axis is temperature-dependent and can be extracted by the measurements of magnetic properties. ${ }^{28}$ With temperature decreasing toward $50 \mathrm{~K}$, these coupling factors can become a non-negligible source of anisotropy. As shown in Fig. 6, the difference in the amplitudes for the response along the $z$ and $x$ axes can become relevant at those temperatures. However, as shown in the inset of Fig. 6, the ratio between the amplitudes along the $x$ and $z$ axes increases with temperature up to an inversion point. Therefore, the source of the anisotropy associated only with dysprosium ions seems not to be effective for the interpretation of the experimental data.

\section{Stable phase at low temperatures}

In this last subsection, we focus on the $\Gamma_{1}$ phase that is stable at low temperatures.

The measurements reported in Ref. 12 show the maximum value of the amplitude of the magnetization between 20 and $50 \mathrm{~K}$. Moreover, the frequency of the photoinduced magnetization is constant in this temperature range at the value close to $50 \mathrm{~K}$ that is characteristic of the $\Gamma_{4}$ phase. Therefore, the laser pulse is inducing excitation modes of the

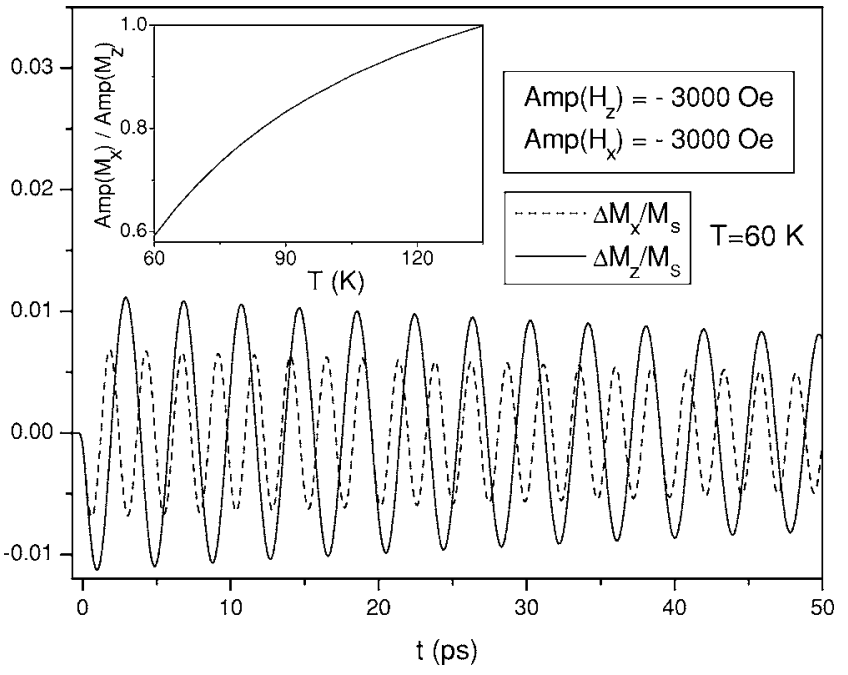

FIG. 6. Comparison between amplitudes for excitations with fields along the $z$ and $x$ axes as a function of time at $T=60 \mathrm{~K}$. Inset: the ratio of these amplitudes as a function of the temperature for times just after the pulse.

phase that should be unstable at low temperatures.

In order to stabilize the $\Gamma_{1}$ phase, the free energy (1) has to be supplemented with a quartic term, ${ }^{28}$ for instance, a term that decreases the energy when the moments are antiferromagnetically aligned along the $y$ axis (see Fig. 1). We assume $V \rightarrow V^{\prime}=V-A_{y y}^{(2)}\left(Y_{1}-Y_{2}\right)^{4}$ in order to describe the system in simple terms. Upon linearization around the equilibrium configuration $Y_{1}^{\mathrm{eq}}=1$ and $Y_{2}^{\mathrm{eq}}=-1$, the two modes characteristic of this phase can be derived. The first one, with frequency

$$
\frac{\omega_{z}^{2}}{\gamma^{2}}=2 E\left(32 A_{y y}^{(2)}-2 A_{x x}\right)-D^{2}
$$

corresponds to a dynamic with $\Delta X_{1}=-\Delta X_{2}$ and $\Delta Z_{1}=\Delta Z_{2}$, while the second mode, with frequency

$$
\frac{\omega_{x}^{2}}{\gamma^{2}}=2 E\left(2 A_{z z}-32 A_{y y}^{(2)}\right)-D^{2},
$$

is characterized by $\Delta X_{1}=\Delta X_{2}$ and $\Delta Z_{1}=-\Delta Z_{2}$. We follow the procedure of Sec. II: the anisotropy constants are derived by Eqs. (34) and (35) and the experimental Raman spectra ${ }^{16}$ by imposing $A_{y y}^{(2)}=0$. At $T=50$, we find $A_{x x}\left(\Gamma_{1}\right)=-1040$ Oe that is close to $A_{x x}\left(\Gamma_{4}\right)=-640$ Oe obtained if the $\Gamma_{4}$ phase is stable at that temperature.

The values of $A_{y y}^{(2)}$ required to stabilize the $\Gamma_{1}$ phase are small. The free energy corresponding to the equilibrium position of the $\Gamma_{1}$ phase is $V_{1}=-E-16 A_{y y}^{(2)}$, while that of the $\Gamma_{4}$ phase is at first order in the canting angle $\beta_{0}$,

$$
V_{4}=-E-D\left(2 \beta_{0}\right)-2 A_{x x}=-E-\frac{D^{2}}{E}-2 A_{x x} .
$$

The condition $V_{1}<V_{4}$ implies that 


$$
A_{y y}^{(2)}>\frac{D^{2}}{16 E}+\frac{A_{x x}}{8} .
$$

Taking into account the values of the exchange fields given in the previous section and the anisotropy constant $A_{x x}\left(\Gamma_{1}\right)$, we derive $A_{y y}^{(2)}>60$ Oe. Therefore, the value of the constant $A_{y y}^{(2)}$ is consistent with the expansion of the free energy being only a fraction of the anisotropy energies $A_{x x}$ and $A_{z z}$.

If the parameter $A_{y y}^{(2)}$ is of the order of hundreds of Oersted, the phases $\Gamma_{4}$ and $\Gamma_{1}$ are close in energy. Due to a small light absorption, the laser pulse could affect the stability of the system by favoring the $\Gamma_{4}$ phase on a short time scale. Therefore, the femtosecond pulse could induce a reorientational phase transition from $\Gamma_{1}$ to $\Gamma_{4}$ state in analogy with the antiferromagnetic-to-ferromagnetic phase transition induced by heating with a laser in FeRh films. ${ }^{29}$ Finally, we point out that a static magnetic field gives rise to a spin reorientational transition in orthoferrites. ${ }^{28}$ Hence the role of the magneticfield pulse induced via the inverse Faraday effect could be investigated in relation to the perturbation of the phase stability. This is left for future investigations.

\section{SUMMARY}

Stimulated by recent experimental results showing ultrafast nonthermal control of magnetization by instantaneous photomagnetic pulses in dysprosium orthoferrites, a theoretical study of magnetization dynamics has been presented in this paper. We have employed a general form of free energy suitable for dysprosium orthoferrites whose parameters are derived from experimental measurements. We have solved coupled sublattice Landau-Lifshitz-Gilbert equations whose damping parameter is determined by considering the scattering rate due to magnon-magnon interaction. Due to the inverse Faraday effect, the magnetic fields perturbing the equilibrium configuration can be modeled as Gaussian pulses with amplitude proportional to the intensity of the light pulse and time width of the order of 100 fs. The nonlinear dynamical equations have been integrated through an optimized Runge-Kutta algorithm, and an analytical solution of the linearized system has been discussed in the case when the magnetic field pulse is assumed to have the shape of a $\delta$ function. This solution provides the right orders of magnitude allowing us to interpret the experimental results in simple terms.

We have found that the quasi-antiferro mode is excited by the pulse along the $z$ axis and the oscillations of the magnetization have amplitudes compatible with experiment. Magnetic fields in opposite directions give rise to out-of-phase oscillations showing a behavior in agreement with experimental results for times longer than the initial transient. We have stressed that the magnetization dynamics is not only strongly influenced by the amplitude of the magnetic field pulse, but also by the parameters determining the free energy, in particular the ratio between the antisymmetric and symmetric exchange energies. The temperature dependence of the magnetization dynamics has been discussed showing that the proposed damping mechanism based on magnonmagnon scattering can be effective on the picosecond scale. When the field pulse is along the $x$ axis, the quasiferro mode is excited, but there are some discrepancies in the comparison between theory and data. We point out that the response along the $x$ axis can be strongly influenced in orthoferrites by several effects, such as the optical birefringence and the anisotropy of the magneto-optical susceptibility. Finally, the behavior of the magnetization has been analyzed in the lowtemperature range where, due to an unavoidable heating effect, the laser pulse could perturb the stability between the $\Gamma_{1}$ and $\Gamma_{4}$ state.

We notice that the model proposed in this work has neglected dipolar contributions because they are orders of magnitude smaller than exchange and anisotropy terms. Moreover, due to the fact that the effective magnetic field obtained through the inverse Faraday effect shows spatial variations negligible on the microscopic scale, only the spin-wave modes at zero wave vector are excited. Since the static magnetization changes slowly in the investigated temperature range, the presence of spin waves with wave vectors different from zero should not provide sizable contributions to the dynamic behavior. Moreover, the experimental samples were in a monodomain state. Therefore, the macrospin approximation employed in this paper can be considered reliable.

Finally, we point out that the approach employed for dysprosium orthoferrites can also be generalized to describe the magnetization dynamics of other rare-earth orthoferrites, at least in the $\Gamma_{4}$ phase. The anisotropy constants are the only quantities strongly dependent on the rare-earth ion, but these do not play a major role in affecting statics and dynamics. On the other hand, the most important values of the exchange fields are of the same order in several rare-earth orthoferrites. ${ }^{16}$ Recently, this approach has been employed in order to understand the possibility of coherent control in orthoferrrites and garnet films via the inverse Faraday effect. ${ }^{30}$ Clearly the approach proposed in this paper is suitable for magnetic dielectrics and not for metallic itinerant magnets. However, up to now, due to the unavoidable light absorption, it has been impossible to ascertain the role played by the inverse Faraday effect on the magnetization dynamics of itinerant magnets.

\section{ACKNOWLEDGMENTS}

We like to thank A. V. Kimel and A. Bringer for a critical reading of the manuscript, and A. Kirilyuk and S. Woodford for useful discussions. C.A.P. acknowledges financial support by the European RTN network DYNAMICS. 
${ }^{1}$ E. Beaurepaire, J.-C. Merle, A. Daunois, and J.-Y. Bigot, Phys. Rev. Lett. 76, 4250 (1996).

${ }^{2}$ J. Hohlfeld, E. Matthias, R. Knorren, and K. H. Bennemann, Phys. Rev. Lett. 78, 4861 (1997).

${ }^{3}$ A. Scholl, L. Baumgarten, R. Jacquemin, and W. Eberhardt, Phys. Rev. Lett. 79, 5146 (1997).

${ }^{4}$ B. Koopmans, M. van Kampen, J. T. Kohlhepp, and W. J. M. de Jonge, Phys. Rev. Lett. 85, 844 (2000).

${ }^{5}$ G. P. Zhang and W. Hübner, Phys. Rev. Lett. 85, 3025 (2000).

${ }^{6}$ L. Guidoni, E. Beaurepaire, and J.-Y. Bigot, Phys. Rev. Lett. 89, 017401 (2002).

${ }^{7}$ G. Ju, J. Hohlfeld, B. Bergman, R. J. M. van de Veerdonk, O. N. Mryasov, J.-Y. Kim, X. Wu, D. Weller, and B. Koopmans, Phys. Rev. Lett. 93, 197403 (2004).

${ }^{8}$ A. V. Kimel, A. Kirilyuk, A. Tsvetkov, R. V. Pisarev, and Th. Rasing, Nature (London) 429, 850 (2004).

${ }^{9}$ J. Hohlfeld, Th. Gerrits, M. Bilderbeek, Th. Rasing, H. Awano, and N. Ohta, Phys. Rev. B 65, 012413 (2002).

${ }^{10}$ A. V. Kimel, A. Kirilyuk, P. A. Usachev, R. V. Pisarev, A. M. Balbashov, and Th. Rasing, Nature (London) 435, 655 (2005).

${ }^{11}$ Van der Ziel, J. P. Pershan, and P. S. Malstrom, Phys. Rev. Lett. 15, 190 (1965).

${ }^{12}$ P. S. Pershan, van der Ziel, and P. S. Malstrom, Phys. Rev. 143, 574 (1966).

${ }^{13}$ P. S. Pershan, J. Appl. Phys. 38, 1482 (1967).

${ }^{14}$ F. Hansteen, A. Kimel, A. Kirilyuk, and Th. Rasing, Phys. Rev. Lett. 95, 047402 (2005); F. Hansteen, A. Kimel, A. Kirilyuk, and Th. Rasing, Phys. Rev. B 73, 014421 (2006).

${ }^{15}$ R. M. White, R. J. Nemanich, and C. Herring, Phys. Rev. B 25, 1822 (1982).
${ }^{16}$ N. Koshizuka and K. Hayashi, J. Phys. Soc. Jpn. 57, 4418 (1988).

${ }^{17}$ G. F. Herrmann, J. Phys. Chem. Solids 24, 597 (1963).

${ }^{18}$ R. L. White, J. Appl. Phys. 40, 1061 (1969).

${ }^{19}$ We have checked that the circularly polarized magnetic field associated with the electromagnetic pulse with frequency corresponding to $1.55 \mathrm{eV}$ gives a contribution to the amplitude of the response that is at least four orders of magnitude less than that obtained when the inverse Faraday effect is active.

${ }^{20}$ A. V. Kimel, R. V. Pisarev, J. Hohlfeld, and Th. Rasing, Phys. Rev. Lett. 89, 287401 (2002).

${ }^{21}$ C. H. Tsang, R. L. White, and R. M. White, J. Appl. Phys. 49, 6063 (1978).

${ }^{22}$ A. H. Morrish, The Physical Principles of Magnetism (John Wiley and Sons, Inc., New York, 1965).

${ }^{23}$ R. K. Nagle and E. B. Saff, Fundamentals of Differential Equations, 2nd ed. (The Benjamin/Cummings Publishing Company, Redwood City, CA, 1989).

${ }^{24}$ A. V. Kimel (private communication).

${ }^{25}$ S. R. Woodford, A. Bringer, and S. Blügel (unpublished).

${ }^{26}$ W. K. Tabor and F. S. Chen, J. Appl. Phys. 40, 2760 (1969).

${ }^{27}$ P. A. Usachev, R. V. Pisarev, A. M. Balbashov, A. V. Kimel, A. Kirilyuk, and Th. Rasing, Phys. Solid State 47, 2292 (2005).

${ }^{28}$ A. K. Zvezdin and V. M. Matveev, Sov. Phys. JETP 50, 543 (1985).

${ }^{29}$ J.-H. Thiele, M. Buess, and C. H. Back, Appl. Phys. Lett. 85, 2857 (2004).

${ }^{30}$ C. A. Perroni and A. Liebsch, J. Phys.: Condens. Matter 18, 7063 (2006). 